\title{
Change in treatment modality and trends in survival among stage I non-small cell lung cancer patients: a population-based study
}

\author{
Gileh-Gol Akhtar-Danesh ${ }^{1}$, Christian Finley ${ }^{2}$, Hsien Yeang Seow $^{3,4}$, Saad Shakeel $^{5}$, Noori Akhtar-Danesh ${ }^{3,6}$ \\ ${ }^{1}$ Department of Surgery, ${ }^{2}$ Division of Thoracic Surgery, ${ }^{3}$ Department of Health Research Methods, Evidence, and Impact, ${ }^{4}$ Department of \\ Oncology, McMaster University, Hamilton, Ontario, Canada; ${ }^{5}$ School of Medicine, University of Toronto, Toronto, Ontario, Canada; ${ }^{6}$ School of \\ Nursing, McMaster University, Hamilton, Ontario, Canada \\ Contributions: (I) Conception and design: GG Akhtar-Danesh, C Finley, N Akhtar-Danesh; (II) Administrative support: N Akhtar-Danesh; (III) \\ Provision of study materials or patients: HY Seow; (IV) Collection and assembly of data: N Akhtar-Danesh; (V) Data analysis and interpretation: N \\ Akhtar-Danesh, GG Akhtar-Danesh, C Finley; (VI) Manuscript writing: All authors; (VIII) Final approval of manuscript: All authors. \\ Correspondence to: Noori Akhtar-Danesh, PhD. School of Nursing, McMaster University, 1280 Main St. West, Room 2J21D, Hamilton, Ontario L8S \\ 4K1, Canada. Email: daneshn@mcmaster.ca.
}

Background: A number of treatment modalities are available to patients with early non-small cell lung cancer (NSCLC) but there is inconsistency regarding their effects on survival. The associated survival of each treatment modality is crucial for patients in making informed treatment decisions. We aimed to examine the change in treatment modality and trends in survival for patients with stage I NSCLC and assess the association between treatment modality and survival.

Methods: All patients diagnosed with stage I NSCLC in the Canadian province of Ontario between 2007 and 2015 were included in this population-based study. We used a flexible parametric model to estimate the trends in survival rate.

Results: Overall, 11,910 patients were identified of which 7,478 patients (62.8\%) received surgical resection and 2,652 (22.3\%) radiation only. The proportion of patients who received radiation only increased from $13.2 \%$ in 2007 to $28.0 \%$ in 2015 (P-for-trend <0.001). Survival increased for all treatment modalities from 2007 to 2015 . The increase in 5 -year survival was more than $20 \%$ for all surgical groups and more than $35 \%$ for radiation-only group.

Conclusions: The survival of patients with stage I NSCLC increased for all treatment modalities over the study period, most distinctly in elderly patients, which coincided with a rise in the use of radiation therapy. While surgical resection was associated with the best chance of 5-year survival, radiation therapy is a safe and effective treatment for medically inoperable patients with early disease.

Keywords: Non-small cell lung cancer (NSCLC); survival analysis; treatment modality; trends in survival

Submitted Mar 18, 2020. Accepted for publication Aug 03, 2020.

doi: $10.21037 /$ jtd-20-1387

View this article at: http://dx.doi.org/10.21037/jtd-20-1387

\section{Introduction}

Lung cancer is the leading cause of cancer-related mortality worldwide (1). Despite representing less than $20 \%$ of all new cancer diagnosis in 2017, its mortality surpassed those of colorectal, breast, and prostate cancer combined (2). The Surveillance, Epidemiology, and End Results (SEER) program estimated that between 2005-2011, approximately
$16 \%$ of lung cancers were diagnosed at stage I (3). The estimated 5-year survival for pathologic stage I non-small cell lung cancer (NSCLC) ranges from $73 \%$ to $90 \%$ based on the $8^{\text {th }}$ edition of the TNM staging system (4). Surgical resection is the treatment of choice for patients with clinical stage I disease who are surgical candidates (5). Lobectomy is the preferred operative approach, with preservation of 
pulmonary function and good oncologic outcomes (6). Adjuvant chemotherapy is subsequently recommended for a small subset of patients with stage IB disease with a high risk of recurrence (7). In patients whose comorbidities preclude surgical resection, radiation therapy can be offered as definitive treatment, with stereotactic body radiation therapy (SBRT) being the preferred approach (8). As it stands, there is a paucity of large-scale studies examining the comparative survival of stage I NSCLC patients based on treatment modality in Canada.

This population-based study aims to examine the changes in treatment modality and evaluate the relevant survival among patients with stage I lung cancer. The analysis is limited to patients with NSCLC, as the prognosis and management of small cell lung cancer is drastically different. We present the following article in accordance with the MDAR reporting checklist (available at http:// dx.doi.org/10.21037/jtd-20-1387).

\section{Methods}

\section{Data source}

The main dataset used in this analysis was the Ontario Cancer Registry (OCR), which includes date of diagnosis, stage, and tumor characteristics for each cancer patient. The OCR includes all diagnosed cases of invasive neoplasia, except basal and squamous cell skin cancers in Ontario (9). We also used several population-based or administrative datasets held at the Institute for Clinical Evaluative Sciences (ICES). These datasets include: Cancer Activity Level Reporting, Discharge Abstracts Database, National Ambulatory Care Reporting System, Ontario Health Insurance Plan Claims, Same Day Surgery datasets, and Registered Person Database. These datasets were linked using unique encoded identifiers and analyzed at ICES. This study includes all Ontario residents diagnosed with primary invasive NSCLC with known stage I disease from 2007 to 2015, with follow-up to the end of 2016.

Using the International Statistical Classification of Diseases and Related Health Problems, Tenth Revision (ICD-10), patients with stage I lung cancer with the codes of C34.0 (malignant neoplasm of main bronchus), C34.1 (malignant neoplasm upper lobe, bronchus or lung), C34.2 (malignant neoplasm of middle lobe, bronchus or lung), C34.3 (malignant neoplasm of lower lobe, bronchus or lung), C34.8 (overlapping malignant lesion of bronchus or lung), and C34.9 (malignant neoplasm of bronchus or lung unspecified) were identified in the OCR dataset. Then, using the International Classification of Diseases for Oncology (ICD-O-2 or ICD-O-3), all cases with small cell carcinoma, carcinoid tumors, salivary gland tumors, mesenchymal tumors, lymphoproliferative tumors, and miscellaneous tumors were excluded (10). Patients were excluded if the diagnosis was based solely on death certificate or autopsy, as the survival time could not be identified for these patients. The analysis included patients 18 years and older at the time of diagnosis. Age at diagnosis was then classified as $<60,60-69,70-79$, and $\geq 80$ years.

Treatment modalities were investigated based on the Canadian Classification of Health Information (CCI) (11) and Canadian Classification of Diagnostic, Therapeutic, and Surgical Procedures (CCP) (12) and were classified as: (I) no-treatment for patients who received no treatment, or with no record of treatment, (II) radiation only (RT) for patients receiving only radiation therapy, (III) chemotherapy with or without radiation (Chemo \pm RT). The surgical groups include: (IV) sublobar resection or lobectomy only (Sub/Lob only) with no record of radiation or chemotherapy, (V) sublobar resection or lobectomy with radiation or chemotherapy (Sub/Lob + RT/Chemo), and (VI) pneumonectomy with or without radiation or chemotherapy (pneumonectomy $\pm \mathrm{RT} /$ Chemo). To guide treatment classifications, a preliminary analysis was conducted to examine the Kaplan-Meier survival curves for all surgical and non-surgical groups. The KaplanMeier curves were adjusted for patient age, stage of disease, and histology of tumor. Although expertise with anatomic segmentectomies is increasing amongst thoracic surgeons, and despite the lower morbidity of less extensive resections (13-15), the Kaplan-Meier curves for sublobaronly and lobectomy-only resections were similar, and were therefore combined into one group (Sub/Lob only). Also, sublobar and lobectomy surgical groups with radiation or chemotherapy were combined into one group (Sub/Lob + RT/Chemo). In addition, video-assisted thoracoscopic surgery (VATS) and open surgery were not analyzed separately. For each patient, a treatment modality was accounted for and included in the analysis only if it was received up to 1 year after diagnosis. The exact sequence of multi-modal therapy was not available.

Staging data has been made available in Ontario since 2007. Cancer Care Ontario uses a best stage grouping approach, where stage is assigned to each case based on pathologic TNM when available, and clinical TNM otherwise (16). It collects stage data using the staging 
criteria of the American Joint Committee on Cancer or the Collaborative Stage initiative. If a case has more than one valid stage value, the "best stage" is derived based on a pre-specified algorithm if a collaborative stage is available; otherwise, the stage group provided by the treating regional cancer centre is used (17).

Tumor histology was classified as: (I) carcinoma-not otherwise specified (NOS), (II) squamous cell carcinoma (SCC), (III) adenocarcinoma (AC), or (IV) other.

\section{Statistical analysis}

First, descriptive measures were reported for each category of variables sex, age group, histology of tumor, and year of diagnosis based on treatment modality. Then, a logistic regression analysis was conducted to quantify the effects of sex, age group, histology of tumor, and year of diagnosis on the chance of receiving surgical resection. For this analysis, treatment modalities were regrouped as surgery and nosurgery (reference group). The surgery group included all surgical resection groups with or without chemotherapy or radiation. The no-surgery group included all the nonsurgical groups. Next, the survival rate was estimated for patients diagnosed with stage I NSCLC from 2007 to 2015 in Ontario with follow-up until the end of 2016. We used a flexible parametric (Royston-Parmar) model $(18,19)$ to estimate the trends in survival based on treatment modality, adjusted for sex, age, year of diagnosis, and histology of tumor. This method uses a piecewise approach in modeling, which is more capable of mimicking the actual trends survival patterns compared to other traditional methods (20). We fitted the model by incorporating age, sex, year of diagnosis, histology of tumour, treatment modality, and the interaction term between each two variables into a multiple statistical model using a forward approach. The likelihood ratio test was used to compare between different models, and to reach to a final model. All variables included in the final model were statistically significant $(\mathrm{P} \leq 0.05)$. Then, based on the final model, trends in 1-, 2-, and 5-year survival rates were estimated for each treatment modality while adjusting for other variables.

The analyses were conducted using Stata/MP 15.1 (Stata Corporation, College Station, TX, USA). The flexible parametric model was fitted using the freely available stpm2 program (18) written for Stata package.

The study was conducted in accordance with the Declaration of Helsinki. The study was approved by institutional ethics board of the Hamilton Integrated
Research Ethics Board (HiREB\# 2921). ICES is a prescribed entity under section 45 of Ontario's Personal Health Information Protection Act. Section 45 authorizes ICES to collect personal health information, without consent, for the purpose of analysis or compiling statistical information with respect to the management of, evaluation or monitoring of, the allocation of resources to or planning for all or part of the health system. Projects conducted under section 45 , by definition, do not require informed consent. This project was conducted under section 45 , and approved by ICES' Privacy and Legal Office.

\section{Results}

Overall, 11,910 patients with stage I NSCLC were identified, of which $53.6 \%(\mathrm{n}=6,384)$ were female. The mean age at diagnosis was $70.9(\mathrm{SD}=9.8)$ years; 71.7 ( $\mathrm{SD}=9.4)$ years for males and $70.2(\mathrm{SD}=10.1)$ years for females. AC was the most common subtype of NSCLC, comprising $34.5 \%$ of cases.

Table 1 summarizes the distribution of sex, age group, histology of tumor, and year of diagnosis based on treatment modality. Overall, 7,478 patients (62.8\%) received surgical resection, 2,652 (22.3\%) radiation only, and 1,172 (9.8\%) did not receive any active treatment. Only 608 (5.1\%) received chemotherapy with or without radiation and only $109(0.9 \%)$ underwent pneumonectomy. We also found differences in treatment modalities between males and females; $60.5 \%$ of males received surgical resection, in contrast to $64.7 \%$ of females $(\mathrm{P}<0.001)$. The older the patients, the more likely they were to receive no treatment or radiation only, with the proportion of no-treatment varying from $4.6 \%$ to $22.7 \%$ between the youngest to oldest age group (Table 1). The proportion of patients who received radiation only changed from $7.0 \%$ from the youngest age group to $40.8 \%$ for the oldest age group.

\section{Changes in treatment modalities}

The distribution of treatment modalities was not the same over the 9 years of the study period. One notable change was the increasing trend in the proportion of radiation only from $13.2 \%$ in 2007 to $28.0 \%$ in 2015 (P-for-trend $<0.001)$. The increasing trend was more pronounced in the oldest age group, where the proportion of patients receiving radiation only increased from $28.7 \%$ in 2007 to $51.2 \%$ in 2015 (P-for-trend <0.001) (Table 2). Table 2 also indicates that the proportion of elderly patients receiving surgical 
Table 1 Distribution of lung cancer based on patient demographics, tumor histology, year of diagnosis, and treatment modality

\begin{tabular}{|c|c|c|c|c|c|c|c|}
\hline Variable & No treatment & RT only & Chemo $\pm \mathrm{RT}$ & Sub/Lob only & Sub/Lob + RT/Chemo & Pneum. \pm RT/Chemo & Total \\
\hline Male & $582(10.5)$ & $1,299(23.5)$ & $300(5.4)$ & $2,598(47.0)$ & $683(12.4)$ & $64(1.2)$ & 5,526 \\
\hline Female & $590(9.2)$ & $1,353(21.2)$ & $308(4.8)$ & $3,320(52.0)$ & $768(12.0)$ & $45(0.7)$ & 6,384 \\
\hline \multicolumn{8}{|l|}{ Age group } \\
\hline 60-69 years & $161(4.7)$ & $470(13.6)$ & $177(5.1)$ & $2,088(60.5)$ & $527(15.3)$ & $26(0.8)$ & 3,449 \\
\hline 70-79 years & $394(8.8)$ & $1,093(24.3)$ & $231(5.1)$ & $2,221(49.4)$ & $517(11.5)$ & $44(1.0)$ & 4,500 \\
\hline$\geq 80$ years & $545(22.7)$ & $981(40.8)$ & $139(5.8)$ & $627(26.1)$ & $106(4.4)$ & $8(0.3)$ & 2,406 \\
\hline Mean (SD) & $77.0(10.1)$ & $75.9(8.5)$ & $71.9(9.4)$ & $68.4(9.1)$ & $67.0(9.2)$ & $66.7(10.3)$ & $70.9(9.8$ \\
\hline SCC & $206(7.5)$ & $672(24.3)$ & $161(5.8)$ & $1,305(47.2)$ & $373(13.5)$ & $45(1.6)$ & 2,762 \\
\hline$A C$ & $252(6.1)$ & $974(23.7)$ & $228(5.6)$ & $2,167(52.8)$ & $458(11.2)$ & $26(0.6)$ & 4,105 \\
\hline Other & $529(14.6)$ & $364(10.0)$ & $80(2.2)$ & $2,145(59.2)$ & $480(13.3)$ & $24(0.7)$ & 3,622 \\
\hline \multicolumn{8}{|c|}{ Year of diagnosis } \\
\hline 2007-2009 & $318(9.5)$ & 555 (16.6) & $178(5.3)$ & $1,720(51.6)$ & $505(15.1)$ & $60(1.8)$ & 3,336 \\
\hline 2010-2012 & $441(11.5)$ & $842(21.9)$ & $189(4.9)$ & $1,894(49.2)$ & 455 (11.8) & $28(0.7)$ & 3,849 \\
\hline 2013-2015 & $413(8.7)$ & $1,255(26.6)$ & 241 (5.1) & $2,304(48.8)$ & $491(10.4)$ & $21(0.4)$ & 4,725 \\
\hline Total & $1,172(9.8)$ & $2,652(22.3)$ & $608(5.1)$ & $5,918(49.7)$ & $1,451(12.2)$ & $109(0.9)$ & 11,910 \\
\hline
\end{tabular}

Carc., carcinoma, NOS; SCC, squamous cell carcinoma; AC, adenocarcinoma; NOS, not otherwise specified; RT, radiation therapy; Chemo, chemotherapy; Sub, sublobar resection; Lob, lobectomy; pneum., pneumonectomy; SD, standard deviation.

resection decreased from $43.1 \%$ in 2007 to $30.9 \%$ in 2015 (P-for-trend $<0.001$ ).

\section{Predictors of surgical resection}

Table 3 presents the predictors of surgical resection using a multiple logistic regression, where the chance of undergoing surgical resection is modeled based on sex, age group, year of diagnosis, and histology of tumor. The results are presented as odds ratio (OR) with $95 \%$ confidence interval (95\% CI). After adjusting for other variables, female patients were $10 \%$ more likely to receive surgical resection compared to male patients (OR 1.10; 95\% CI: 1.01-1.19). As expected, the OR for undergoing surgical resection for patients $\geq 60$ significantly decreased compared to patients $<60$ years; The OR for patients $70-79$ and $\geq 80$ years were 0.31 (95\% CI: $0.26-0.36$ ) and 0.08 (95\% CI: $0.07-0.10$ ), respectively. The chance of surgical resection for patients with SCC and AC tumors was 4.29 (95\% CI: 3.70-4.97) and 4.18 (95\% CI: 3.64-4.81) times higher compared to patients with carcinoma NOS.

\section{Trends in survival rate based on treatment modality}

The final survival model included sex, age group, treatment modality, histology of tumor, year of diagnosis, interaction term between age group and year of diagnosis, and interaction term between treatment modality and year of diagnosis. The model also included treatment modality and year of diagnosis as time dependent variables. Based on the final model, trends in 1-, 2-, and 5-year survival rates from 2007 to 2015 for each treatment modality were estimated (Figure 1). In general, survival increased for all treatment modalities from 2007 to 2015, including for the no treatment group. The increase was more pronounced for the radiation only group and chemotherapy (with or without 
Table 2 Trends of treatment modalities in elderly patients $(80+)$ over the study period (P-for-trend for radiation only group $<0.001)$

\begin{tabular}{|c|c|c|c|c|c|}
\hline Year of diagnosis & No treatment & RT only & Chemo $\pm \mathrm{RT}$ & Surgery groups & Total \\
\hline 2008 & 49 (22.8) & $69(32.1)$ & $17(7.9)$ & $80(37.2)$ & 215 \\
\hline 2009 & $71(30.1)$ & 76 (32.2) & $10(4.2)$ & 79 (33.5) & 236 \\
\hline 2010 & $64(27.0)$ & $73(30.8)$ & $15(6.3)$ & 85 (35.9) & 237 \\
\hline 2012 & $79(27.6)$ & $106(37.1)$ & $17(5.9)$ & $84(29.4)$ & 286 \\
\hline 2013 & $78(23.9)$ & $160(49.1)$ & $13(4.0)$ & $75(23.0)$ & 326 \\
\hline 2014 & $69(20.8)$ & $154(46.4)$ & $21(6.3)$ & $88(26.5)$ & 332 \\
\hline 2015 & $42(12.7)$ & 169 (51.2) & $17(5.2)$ & $102(30.9)$ & 330 \\
\hline
\end{tabular}

$\mathrm{RT}$, radiation therapy; Chemo, chemotherapy.

Table 3 Predictors of surgical resection among patients diagnosed with stage I non-small cell lung cancer

\begin{tabular}{lc}
\hline Variable & OR $(95 \% \mathrm{Cl})$ \\
\hline Sex & Reference \\
Male & $1.10(1.01,1.19)^{+}$ \\
Female & Reference $^{+}$ \\
Age group & $0.61(0.52,0.72)^{++}$ \\
$<60$ years & $0.31(0.26,0.36)^{++}$ \\
$60-69$ years & $0.08(0.07,0.10)^{++}$ \\
$70-79$ years & $0.91(0.90,0.93)^{++}$ \\
$\geq 80$ years & \\
Year of diagnosis & Reference \\
Histology & $4.29(3.70,4.97)^{++}$ \\
Carcinoma, NOS & $4.18(3.64,4.81)^{++}$ \\
SCC & $6.52(5.64,7.54)^{++}$ \\
AC & \\
Other & P<0.05; ${ }^{++}$, P<0.001. SCC, squamous cell carcinoma; AC, \\
adenocarcinoma; NOS, not otherwise specified; OR, odds ratio; \\
Cl, confidence interval.
\end{tabular}

radiation) group, although the last group included only $5.1 \%$ of patients. We observed more than $20 \%$ increase in 2-year survival for the radiation only group and close to $30 \%$ increase for the chemotherapy group. The increase in
5 -year survival was more than $20 \%$ for all surgical groups, more than $35 \%$ for radiation group, and close to $30 \%$ for the chemotherapy group.

Trends in fully adjusted survival rates for age group and sex are shown in Figure 2. The trends were similar for all age groups, although a higher survival was evident for women across all age groups and the increase in survival over time was higher as the age group increased.

\section{Overall 5-year survival rate}

In general, the highest 5-year survival rates were observed for the Sub/Lob only group, followed by the Sub/Lob + RT/Chemo group (Table 4). In comparison, the lowest survival rates were observed for the no-treatment group and chemotherapy (with or without radiation) group. Patients with AC and the group classified as Other had the highest survival rates.

\section{Discussion}

This large population-based study of patients with stage I NSCLC found a shift in treatment modalities from 2007 to 2015 , and specifically a drastic change in the treatment of elderly patients. This study found a significant increase in the use of radiation. In 2007, $13.2 \%$ of patients with stage I disease were treated with radiation, in contrast to $28.0 \%$ in 2015 . This increase was most pronounced in the most elderly group of patients, in whom $28.7 \%$ received radiation in 2007 , compared to $51.2 \%$ in 2015 . Notably, 

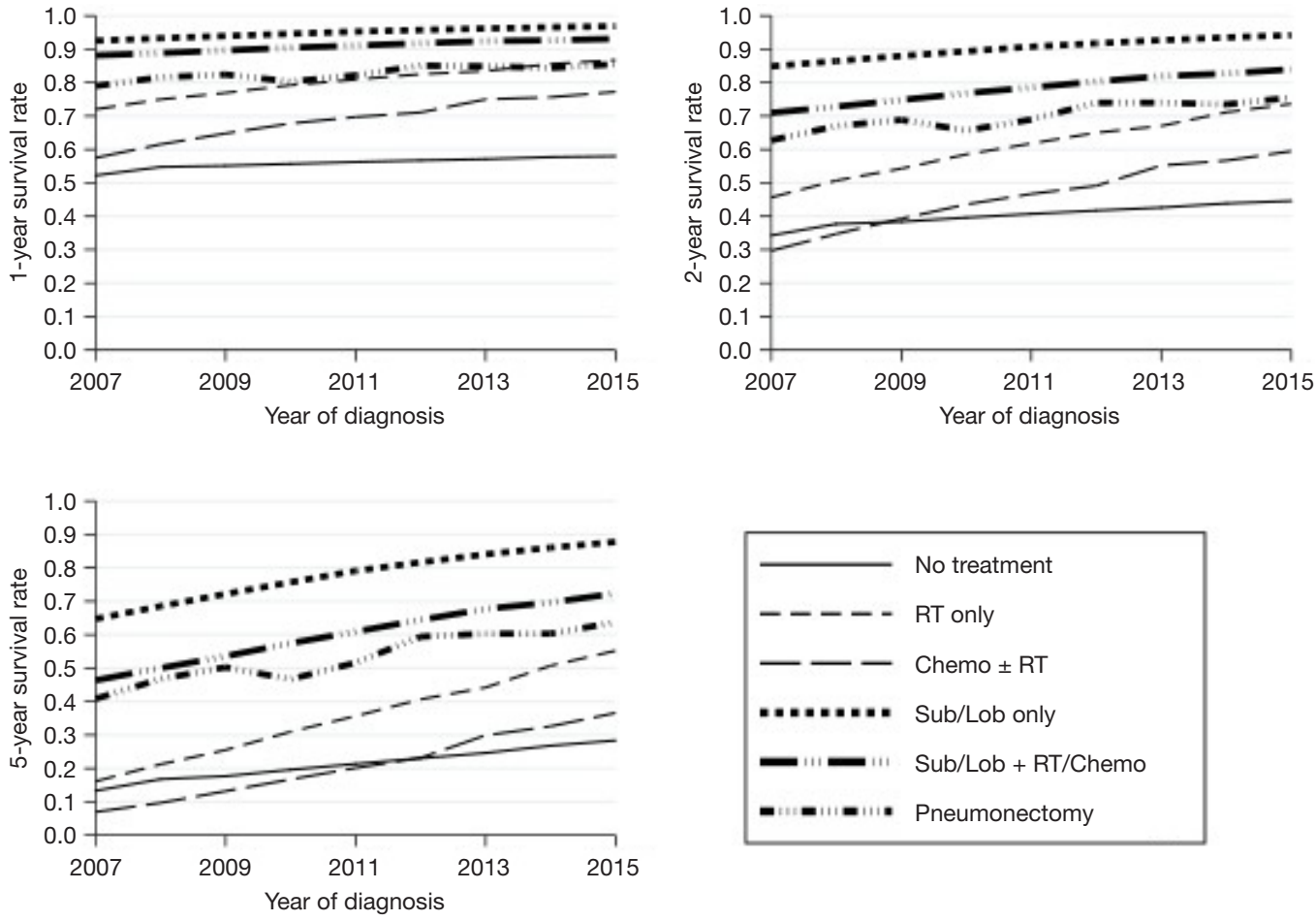

Figure 1 Trends in 1-, 2-, and 5-year survival rates based on treatment modality. The graph is based on the final model which was adjusted for sex, age group, histology of tumor, and year of diagnosis. RT, radiation therapy; Chemo, chemotherapy; Sub, sublobar resection; Lob, lobectomy.
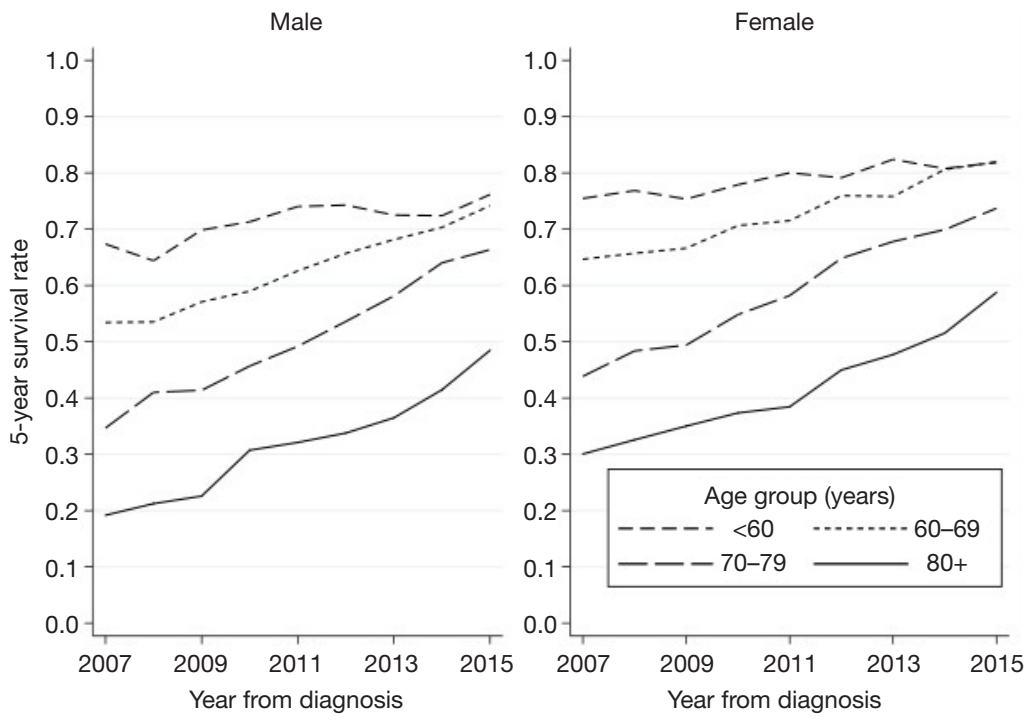

Figure 2 Trends in 5-year survival rate based on sex and age group. The graph is based on the final model which was adjusted for treatment modality, histology of tumor, and year of diagnosis. 
Table 4 Five-year survival rate (95\% CI) for stage I NSCLC based on histology, and treatment modality

\begin{tabular}{lccccccc}
\hline Histology & No treatment & RT only & Chemo \pm RT & Sub/Lob only & $\begin{array}{c}\text { Sub/Lob }+ \\
\text { RT/Chemo }\end{array}$ & $\begin{array}{c}\text { Pneum. } \pm \\
\text { RT/Chemo }\end{array}$ \\
\hline Carc. & $0.17(0.16,0.18)$ & $0.33(0.32,0.34)$ & $0.18(0.16,0.20)$ & $0.75(0.74,0.76)$ & $0.56(0.54,0.59)$ & $0.50(0.43,0.57)$ & $0.41(0.39,0.42)$ \\
SCC & $0.16(0.15,0.18)$ & $0.35(0.34,0.36)$ & $0.16(0.14,0.17)$ & $0.72(0.71,0.73)$ & $0.52(0.51,0.54)$ & $0.43(0.39,0.47)$ & $0.52(0.52,0.53)$ \\
AC & $0.26(0.24,0.27)$ & $0.45(0.44,0.46)$ & $0.29(0.27,0.31)$ & $0.80(0.80,0.81)$ & $0.64(0.63,0.65)$ & $0.57(0.52,0.61)$ & $0.64(0.63,0.64)$ \\
Other & $0.24(0.23,0.25)$ & $0.48(0.47,0.49)$ & $0.24(0.21,0.27)$ & $0.82(0.81,0.82)$ & $0.64(0.63,0.65)$ & $0.58(0.53,0.63)$ & $0.66(0.65,0.67)$ \\
Total & $0.22(0.21,0.22)$ & $0.40(0.39,0.40)$ & $0.22(0.21,0.23)$ & $0.79(0.78,0.79)$ & $0.60(0.60,0.61)$ & $0.50(0.48,0.53)$ & $0.59(0.59,0.60)$ \\
\hline
\end{tabular}

Carc., carcinoma, NOS, SCC, squamous cell carcinoma; AC, adenocarcinoma; RT, radiation therapy; Chemo, chemotherapy; Sub, sublobar resection; Lob, lobectomy; pneum., pneumonectomy; $\mathrm{Cl}$, confidence interval; NSCLC, non-small cell lung cancer.

the rates of surgical resection also decreased in the oldest age group, from $43.1 \%$ to $30.9 \%$. The increase in radiation therapy was also associated with a decrease in the number of patients undergoing pneumonectomy.

Radiation has typically been offered to patients who decline surgery, or who are poor operative candidates, with peripherally located tumours (8). However, with advances in radiation techniques, specifically with the introduction of SBRT, radiation therapy has become an acceptable alternative treatment in a wider range of clinical scenarios (21). The American Society for Radiation Oncology (ASTRO) guidelines suggest that SBRT, with dose modification, can also be an appropriate therapy for patients with centrally located tumours, which may account for the observed decrease in patients undergoing pneumonectomy. Of note, only a small percentage of patients in this study underwent pneumonectomy, which is expected in patients with stage I disease. The most recent ASTRO guidelines also emphasize the important role of radiation therapy in medically inoperable patients (21). This study highlights the uptake of these guidelines in Ontario and shows that the increased use of radiation is also associated with a trend in improved overall survival. A previous study from the Canadian province of Alberta highlighted that access to radiation therapy improves cancer outcomes, even in stage III disease (22).

The increase in survival was most pronounced for the older age groups (Figure 2), whereas survival was relatively steady for the youngest age group. This is likely attributable to the change in treatment of elderly patients. From 2007 to 2015 , the proportion of elderly patients receiving no treatment decreased, as did the proportion receiving surgery, while the use of radiation therapy in this group increased. This suggests that the improving survival of elderly patients with stage I NSCLC is attributable to the fact that these patients were actually receiving treatment with curative intent, namely radiation therapy. Elderly patients have typically had poor survival from lung cancer due to a lack of treatment. This is due to a combination of factors including comorbid illness, frailty, and a perceived lack of benefit with treatment (23). Based on a systematic review of under-treatment in older patients, a vast range of factors were identified that may result in under-treatment (24). For instance, treatment options for older adults are highly affected by physician preferences $(25,26)$, which results in the provision of less aggressive or less effective therapy in older patients (24). In addition to the suboptimal treatment options for the primary tumor, older patients also received differing treatments for the adverse effects of the anticancer drugs, palliative care, pain management, and reconstructive surgery (24). However, the proportion of untreated elderly patients has decreased with the introduction of SBRT. Two recent studies from the Netherlands showed that elderly patients were increasingly undergoing curative therapy since the introduction of SBRT $(27,28)$. These studies also showed an associated increase in the overall survival of elderly patients, with no change in rates of operative intervention.

Finally, patients undergoing surgical resection in the form of a lobar or sublobar resection had the highest rate of 5-year survival. Surgery, by way of a lobectomy, remains the standard of care for medically operable patients with early stage lung cancer (5). Randomized trials are underway comparing the efficacy of lobar versus sublobar resections in early stage disease (15), however, previous studies have suggested worse overall survival for sublobar resections, which is favoured to be attributable to an inadequate lymphadenectomy (13). The increased survival of lobar and sublobar resections compared to other forms of resection is likely attributable to the increased 
morbidity and mortality associated with more extensive resections (29). The comparative benefits of surgery versus SBRT in the treatment of early stage lung cancer are still under investigation (30); however, the comparison of these two modalities was beyond the scope of the present study. The improvement in overall survival over time is favoured to be due to a combination of factors, including the increasing treatment of elderly patients, as well as the increased uptake of minimally-invasive operative techniques (31). Previous studies have shown increased overall survival, and lower operative morbidity associated with VATS (32). Furthermore, advances in chemotherapy, including the introduction of targeted therapies such as epidermal growth factor receptor-tyrosine-kinase inhibitors (EGFR-TKIs), has been shown to have dramatically improved the survival of NSCLC patients (33), though within the context of this study only a small proportion of patients underwent chemotherapy, due to early stage disease. Finally, improvements in staging techniques including the increased uptake of positron emission tomography/computed tomography (PET/CT) and endobronchial ultrasound (EBUS) have allowed for more accurate staging and a decrease in postoperative stage migration, subsequently improving stage-based survival $(34,35)$. Therefore, improvements in both surgical and nonsurgical management of early stage lung cancer are likely responsible for the improved overall survival of patients with stage I disease.

The strengths of this study include the use of a highquality population-based dataset and long follow-up. The ability to report 1- and 2-, and 5-year survival rates provides more insight into the survival of stage I lung cancer patients. Also, the use of a flexible parametric model for analysis allows for a more flexible and powerful approach in mimicking the actual trends in survival patterns $(20,36)$. This study also has several limitations. A subset of patients with stage I disease may have not been captured due to incomplete submission of cancer staging data. Due to the retrospective nature of the study, some factors that may have influenced treatment decisions (including comorbidity status and patient preference) could not be accounted for. Information regarding mediastinal lymphadenectomy was not available, and although pathologic staging was used where possible, this would not have been available for patients undergoing radiation, meaning that these patients may have been down-staged based on their initial clinical staging. Finally, this study was conducted within a singlepayer healthcare system in Ontario and its findings may not be generalizable to private healthcare systems.

In conclusion, we found a shift in treatment modalities offered to patients with stage I NSCLC over time, and more specifically a change in the treatment of elderly patients. Although the overall survival of patients with stage I disease improved over the study period, this improvement was most pronounced for elderly patients, suggesting that this group of patients is increasingly receiving curative therapy. However, for patients fit enough to undergo surgery, the role of radiation, specifically SBRT, is still under investigation and resection remains the treatment modality of choice for stage I disease.

\section{Acknowledgments}

Funding: This study was supported by the Institute for Clinical Evaluative Sciences (ICES), which is funded by an annual grant from the Ontario Ministry of Health and Long-Term Care (MOHLTC). The Ontario Institute for Cancer Research paid for data linkage between different databases. The opinions, results and conclusions reported in this paper are those of the authors and are independent from the funding sources. No endorsement by ICES or the Ontario MOHLTC is intended or should be inferred. Parts of this material are based on data and/or information compiled and provided by CIHI. However, the analyses, conclusions, opinions and statements expressed in the material are those of the authors, and not necessarily those of CIHI. Also, parts of this material are based on data and information provided by Cancer Care Ontario (CCO). The opinions, results, view, and conclusions reported in this paper are those of the authors and do not necessarily reflect those of CCO. No endorsement by CCO is intended or should be inferred. We are indebted to ICES staff at McMaster University, specifically, to Richard Perez and Joshua Cerasuolo, for their invaluable support.

\section{Footnote}

Reporting Checklist: All authors have completed the MDAR reporting checklist. Available at http://dx.doi.org/10.21037/ jtd-20-1387

Peer Review File: Available at http://dx.doi.org/10.21037/jtd20-1387

Conflicts of Interest: All authors have completed the ICMJE uniform disclosure form (available at http://dx.doi. 
org/10.21037/jtd-20-1387). The authors have no conflicts of interest to declare.

Ethical Statement: The authors are accountable for all aspects of the work in ensuring that questions related to the accuracy or integrity of any part of the work are appropriately investigated and resolved. The study was conducted in accordance with the Declaration of Helsinki (as revised in 2013). The study was approved by institutional ethics board of the Hamilton Integrated Research Ethics Board (HiREB\# 2921). ICES is a prescribed entity under section 45 of Ontario's Personal Health Information Protection Act. Section 45 authorizes ICES to collect personal health information, without consent, for the purpose of analysis or compiling statistical information with respect to the management of, evaluation or monitoring of, the allocation of resources to or planning for all or part of the health system. Projects conducted under section 45, by definition, do not require informed consent. This project was conducted under section 45, and approved by ICES' Privacy and Legal Office.

Open Access Statement: This is an Open Access article distributed in accordance with the Creative Commons Attribution-NonCommercial-NoDerivs 4.0 International License (CC BY-NC-ND 4.0), which permits the noncommercial replication and distribution of the article with the strict proviso that no changes or edits are made and the original work is properly cited (including links to both the formal publication through the relevant DOI and the license). See: https://creativecommons.org/licenses/by-nc-nd/4.0/.

\section{References}

1. International Agency for Research on Cancer (IARC). World Cancer Report 2014. Lyon, France: IARC, 2014.

2. Canadian Cancer Society's Advisory Committee on Cancer Statistics. Canadian Cancer Statistics 2017. Toronto, ON: Canadian Cancer Society, 2017.

3. National Cancer Institute. SEER Cancer Statistics Review, 1975-2012, Lung and Bronchus. National Cancer Institute, 2015.

4. Goldstraw P, Chansky K, Crowley J, et al. The IASLC Lung Cancer Staging Project: Proposals for Revision of the TNM Stage Groupings in the Forthcoming (Eighth) Edition of the TNM Classification for Lung Cancer. J Thorac Oncol 2016;11:39-51.

5. National Comprehensive Cancer Network. NCCN
Clinical Practice Guidelines in Oncology: Non-Small Cell Lung Cancer. NCCN, 2019.

6. Ginsberg RJ, Rubinstein LV. Randomized trial of lobectomy versus limited resection for T1 N0 non-small cell lung cancer. Lung Cancer Study Group. Ann Thorac Surg 1995;60:615-22; discussion 622-3.

7. Pignon JP, Tribodet H, Scagliotti GV, et al. Lung adjuvant cisplatin evaluation: a pooled analysis by the LACE Collaborative Group. J Clin Oncol 2008;26:3552-9.

8. Schneider BJ, Daly ME, Kennedy EB, et al. Stereotactic Body Radiotherapy for Early-Stage Non-Small-Cell Lung Cancer: American Society of Clinical Oncology Endorsement of the American Society for Radiation Oncology Evidence-Based Guideline. J Clin Oncol 2018;36:710-9.

9. Cancer Care Ontario. Ontario Cancer Registry. 2018. Available online: https://www.cancercareontario.ca/en/ cancer-care-ontario/programs/data-research/ontariocancer-registry. Accessed 16 October 20182018.

10. Parkin M, Samet J, Tyczynski JE, et al. Lung cancer epidemiology and etiology. In: Travis WD, Brambilla E, Muller-Hermelink HK et al. editors. World Health Organization Classification of Tumours: Pathology \& Genetics, Tumours of the Lung, Pleura, Thymus, and Heart. Geneva, Switzerland: WHO Press, 2004.

11. Canadian Institute for Health Information. Canadian Classification of Health Interventions. Canadian Institute for Health Information, Ottawa, Ontario. 2015. Available online: https://www.cihi.ca/sites/default/files/cci_volume_ three_2015_en_0.pdf. Accessed 1 June 2018.

12. Statistics Canada. Canadian Classification of Diagnostic, Therapeutic, and Surgical Procedures. Ottawa, Ontario: Statistics Canada, 1986.

13. Khullar OV, Liu Y, Gillespie T, et al. Survival After Sublobar Resection versus Lobectomy for Clinical Stage IA Lung Cancer: An Analysis from the National Cancer Data Base. J Thorac Oncol 2015;10:1625-33.

14. Lv X, Cao J, Dai X, et al. Survival rates after lobectomy versus sublobar resection for early-stage right middle lobe non-small cell lung cancer. Thorac Cancer 2018;9:1026-31.

15. Yang F, Sui $X$, Chen $X$, et al. Sublobar resection versus lobectomy in Surgical Treatment of Elderly Patients with early-stage non-small cell lung cancer (STEPS): study protocol for a randomized controlled trial. Trials 2016;17:191.

16. Booth CM, Li G, Zhang-Salomons J, et al. The impact of socioeconomic status on stage of cancer at diagnosis and survival: a population-based study in Ontario, Canada. 
Cancer 2010;116:4160-7.

17. Ginsburg OM, Fischer HD, Shah BR, et al. A populationbased study of ethnicity and breast cancer stage at diagnosis in Ontario. Curr Oncol 2015;22:97-104.

18. Lambert PC, Royston P. Further development of flexible parametric models for survival analysis. Stata J 2009;9:265-90.

19. Royston P, Parmar MK. Flexible parametric proportional-hazards and proportional-odds models for censored survival data, with application to prognostic modelling and estimation of treatment effects. Stat Med 2002;21:2175-97.

20. Rutherford MJ, Dickman PW, Lambert PC. Comparison of methods for calculating relative survival in populationbased studies. Cancer Epidemiol 2012;36:16-21.

21. Videtic GMM, Donington J, Giuliani M, et al. Stereotactic body radiation therapy for early-stage non-small cell lung cancer: Executive Summary of an ASTRO Evidence-Based Guideline. Pract Radiat Oncol 2017;7:295-301.

22. Liu HW, Kerba M, Lim G, et al. Factors Associated With the Use of Radiation Therapy in Patients With Stage III Non-small Cell Lung Cancer in Alberta, Canada: A Population-based Study. Cureus 2016;8:e851.

23. Sigel K, Bonomi M, Packer S, et al. Effect of age on survival of clinical stage I non-small-cell lung cancer. Ann Surg Oncol 2009;16:1912-7.

24. Bouchardy C, Rapiti E, Blagojevic S, et al. Older female cancer patients: importance, causes, and consequences of undertreatment. J Clin Oncol 2007;25:1858-69.

25. Mandelblatt JS, Hadley J, Kerner JF, et al. Patterns of breast carcinoma treatment in older women: patient preference and clinical and physical influences. Cancer 2000;89:561-73.

26. Silliman RA, Troyan SL, Guadagnoli E, et al. The impact of age, marital status, and physician-patient interactions on the care of older women with breast carcinoma. Cancer
1997;80:1326-34.

27. Palma D, Visser O, Lagerwaard FJ, et al. Impact of introducing stereotactic lung radiotherapy for elderly patients with stage I non-small-cell lung cancer: a population-based time-trend analysis. J Clin Oncol 2010;28:5153-9.

28. Haasbeek CJ, Palma D, Visser O, et al. Early-stage lung cancer in elderly patients: a population-based study of changes in treatment patterns and survival in the Netherlands. Ann Oncol 2012;23:2743-7.

29. Booton R, Jones M, Thatcher N. Lung cancer 7: management of lung cancer in elderly patients. Thorax 2003;58:711-20.

30. Subramanian MP, Meyers BF. Surgical Resection Versus Stereotactic Body Radiation Therapy for Stage I NSCLC: Can Randomized Trials Provide the Solution? Cancers (Basel) 2018;10:310.

31. Detterbeck F, Molins L. Video-assisted thoracic surgery and open chest surgery in lung cancer treatment: present and future. J Vis Surg 2016;2:173.

32. Cai YX, Fu XN, Xu QZ, et al. Thoracoscopic lobectomy versus open lobectomy in stage I non-small cell lung cancer: a meta-analysis. PLoS One 2013;8:e82366.

33. Milovancev A, Stojsic V, Zaric B, et al. EGFR-TKIs in adjuvant treatment of lung cancer: to give or not to give? Onco Targets Ther 2015;8:2915-21.

34. Heineman DJ, Daniels JM, Schreurs WH. Clinical staging of NSCLC: current evidence and implications for adjuvant chemotherapy. Ther Adv Med Oncol 2017;9:599-609.

35. Saji H, Kojima K, Nakamura H. Improved lymph node staging in early-stage lung cancer in the national cancer database: commentary. J Thorac Dis 2018;10:S1092-5.

36. Dickman PW, Lambert PC, Coviello E, et al. Estimating net survival in population-based cancer studies. Int J Cancer 2013;133:519-21.
Cite this article as: Akhtar-Danesh GG, Finley C, Seow HY, Shakeel S, Akhtar-Danesh N. Change in treatment modality and trends in survival among stage I non-small cell lung cancer patients: a population-based study. J Thorac Dis 2020;12(9):46704679. doi: $10.21037 /$ jtd-20-1387 